\title{
A Physical Explanation for Particle Spin
}

\author{
Dirk J. Pons ${ }^{1 *}\left(\mathbb{0}\right.$, Arion D. Pons $^{2}$ (), Aiden J. Pons ${ }^{3}$ \\ ${ }^{1}$ Department of Mechanical Engineering, University of Canterbury, Christchurch, New Zealand \\ ${ }^{2}$ University of Cambridge, Cambridge, UK \\ ${ }^{3}$ University of Canterbury, Christchurch, New Zealand \\ Email: ^dirk.pons@canterbury.ac.nz, arion.pons@gmail.com, aidenpons@gmail.com
}

How to cite this paper: Pons, D.J., Pons, A.D. and Pons, A.J. (2019) A Physical Explanation for Particle Spin. Journal of Modern Physics, 10, 835-860. https://doi.org/10.4236/jmp.2019.107056

Received: May 3, 2019

Accepted: June 22, 2019

Published: June 25, 2019

Copyright $\odot 2019$ by author(s) and Scientific Research Publishing Inc. This work is licensed under the Creative Commons Attribution International License (CC BY 4.0).

http://creativecommons.org/licenses/by/4.0/

\section{cc) (i) Open Access}

\begin{abstract}
CONTEXT The spin of a particle is physically manifest in multiple phenomena. For quantum mechanics (QM), spin is an intrinsic property of a point particle, but an ontological explanation is lacking. In this paper we propose a physical explanation for spin at the sub-particle level, using a non-local hidden-variable (NLHV) theory. APPROACH Mechanisms for spin were inferred from the Cordus NLHV theory, specifically from theorised structures at the sub-particle level. RESULTS Physical geometry of the particle can explain spin phenomena: polarisation, Pauli exclusion principle (Einstein-Podolsky-Rosen paradox), excited states, and selective spin of neutrino species. A quantitative derivation is provided for electron spin $g$-factor $g=2$, and a qualitative explanation for the anomalous component. IMPLICATIONS NLHV theory offers a candidate route to new physics at the sub-particle level. This also implies philosophically that physical realism may apply to physics at the deeper level below QM. ORIGINALITY The electron g-factor has been derived using sub-particle structures in NLHV theory, without using quantum theory. This is significant as the g-factor is otherwise considered uniquely predicted by QM. Explanations are provided for spin phenomena in terms of physical sub-structures to the particle.
\end{abstract}

\section{Keywords}

Non-Local Hidden-Variable Theory, Cordus, g Factor, Fundamental Physics, Spin

\section{Introduction}

The spin of a particle is a key concept for particle physics, and is physically manifest in multiple phenomena such as entanglement, [1] [2] [3] [4] Pauli pairs, photon polarisation, superconductivity, selective spin of neutrino species, 
and electron spin g-factor [5]. Despite spin having physical effects, it has no ontological explanation. Quantum mechanics (QM) instead treats it as an intrinsic parameter. This paper offers a physical explanation for spin using non-local hidden-variable (NLHV) theory, specifically the variant called the Cordus theory [6]. It is shown that, under the assumptions of this theory, spin parameters arise naturally as properties of the physical structures at the sub-particle level. This is then used to provide physical explanations of the Pauli Exclusion Principle and the selective spin of neutrino species. The electron g-factor is derived and it is shown quantitatively that $g=2$ for this NLHV theory.

\section{Context: Orbital and Spin Angular Momentum}

\subsection{Background}

In classical mechanics angular momentum is rotation of a body around an axis. The classical regime gives way to the Fermi-Dirac probability distribution when the separation between particles is much smaller than the de Broglie wavelength of the particles.

In particle physics there are two types of angular momentum, orbital and spin. The sum of orbital angular momentum and spin is the total angular momentum for the particle. The total is conserved, though momentum can be transferred between the orbital and spin components, hence spin-orbit interaction.

The orbital angular momentum is generally believed to involve a particle moving in a circular locus, such as an electron moving round the nucleus, or two quarks spinning around each other. It is quantized, as opposed to being a continuous variable, and takes on integer values. The spin angular momentum (or simply 'spin') is analogous to a quantized rotation of the particle, e.g. the electron, about its own axis. Quantum mechanics disfavors an interpretation of rotation, and instead considers spin to be an intrinsic property. QM also rejects the idea that spin could arise from smaller internal particles rotating around a spin axis, as in a bag or plum-pudding model. The confirmatory evidence appears in the electron spin g-factor. At the particle level the quantum spin is measured with respect to a direction set by the observer, and the outcomes are represented by probabilities of finding the particle with spin in that direction (projection). Under QM the particle has no physical orientation either.

Spin is a vector with a total value and a direction. The fermions take spin values of odd half-integer increments $(1 / 2,3 / 2,5 / 2$, etc.). The spin of the electron, proton, and neutron is $1 / 2$ and this applies to the leptons and quarks generally. These particles are subject to the Pauli Exclusion principle, that two co-located particles are unable to be in the same spin state and instead take different spin directions, e.g. $+1 / 2$ and $-1 / 2$ to achieve this. In contrast bosons have integer spin. These include the photon, mesons (quark plus antiquark), and Higgs boson. These bosons follow Bose-Einstein statistics, i.e. there is no interaction between multiple particles, and they can co-locate. It also applies to some atoms, hence condensed states of $2 \mathrm{He} 2$ in superfluidity, and electron Cooper-pairs in 
superconductivity. Atomic nuclei with even mass number have integer spin, and odd have $1 / 2$ spin.

These attributes of particles are well quantified but no deeper explanation is available.

\subsection{Application}

Spin is an important property that is often used in entanglement experiments [1] [2] [3] [4] and interferometry [7]. It is also a key feature in the further development of quantum theory, e.g. the Higgs mechanism and muon properties. The table of nuclides also shows that spin is key to understanding the properties of the nucleus [8]. Other phenomena where spin is important are annihilation [9] [10] [11], including of muons [12], proton size anomaly [13], and aspects of cosmology such as leptogenesis [14]. Spin is also practically important in optical tweezers [15].

\subsection{Ontological Challenges}

There are several conceptual problems with spin. The first is explaining how spin arises at the fundamental level, why particles have the values they do, and what underpins the Pauli Exclusion principle and Bose-Einstein behavior. For example, QM does not offer any deeper explanation of why spin numbers prevent fermions from co-locating.

A second problem is the lack of explanation for why the type of assembly of particles should affect the spin. For example, individual electrons are fermions, whereas a pair of electrons (Cooper pair) is a boson. Likewise, why are nuclides with odd total of nucleons fermions, while those with even totals are bosons?

Third, there is no satisfactory explanation why multiple $2 \mathrm{He} 2$ nuclei and Cooper-pairs do not also physically co-locate like the photons. They do not contract to a singularity. Given that all are bosons, one expects a consistent behaviour from the same spin property. A related question is why should spin be exclusively $1 / 2$ for elementary fermions, yet merely predominately 1 for bosons? What is the basis of this differentiation?

A fourth issue is that, though quantized, the spin of a particle is nonetheless functionally linked to classical angular momentum, as shown in the empirical Einstein-de Haas effect (an electric current in a coil causes a magnet to rotate), and the complementary Barnett effect (an object becomes magnetized when spun). Why is this?

\subsection{Contrasting Perspectives}

It is undisputed that the spin property can be formalized within quantum mechanics. However QM does not provide an ontological explanation of how these behaviors arise. Attempts to provide physical interpretation have been undertaken from the outset of quantum theory, e.g. by Dirac [5], but spin remains resistant to such description within QM. It is generally accepted that there is no 
explanation, that the properties are merely intrinsic, that $\mathrm{QM}$ is complete as a theory as it is, e.g. [16].

It is understandable that QM would construct spin this way. After all QM is premised on particles being zero-dimensional points, hence internal structures are disallowed. Nonetheless, from a NLHV perspective there ought in principle be an underlying mechanics or sub-structure to the particle, but in practice such explanations have been elusive. While the simpler classes of NLHV theory have been excluded by the Bell-type inequalities [17] [18] [19], it has not been possible to rule them all out [20]. Hence it is not impossible that an NLHV theory may provide a solution, but in practice it has been difficult to find workable variants, and hence this branch of physics has become obscure. Historically the main NLHV theory was the de Broglie-Bohm [21] [22]. There has continued to be interested therein [23] [24] [25], but it has not yet progressed to a comprehensive theory of physics. More recently the Cordus variant of NLHV theory [6] has been shown to explain multiple physical phenomena.

\subsection{Brief Summary of the Cordus Theory}

The Cordus theory [6] postulates the existence of specific physical structures at the sub-particle level, and functional behaviors thereof. Philosophically the theory is premised on physical realism that observable phenomena have underlying physical mechanisms [26]. Design principles were used to construct the theory, hence the features are not arbitrary conjectures [6]. The theory predicts that particles have internal structures and externally emitted discrete forces [5] [26]. See Figure 1 for the predicted structure of the electron, and Figure 2 for the photon.

The figures show the sub-strutures and internal mechanisms that are inferred for these particles, and a brief explanation follows based on [27] [28]. The particle is proposed to have an inner structure comprising two reactive ends, which are a small finite distance apart (span), and each behaves like a particle in their interaction with the external environment, when they are energized. The reactive ends are proposed to emit discrete forces, which react with external fields, matter, and photons. A fibril joins the reactive ends and provides instantaneous connectivity and synchronicity between the two reactive ends. It is a persistent and dynamic structure but does not interact with matter. There is also an external structure whereby the reactive ends periodically energize (at the de Broglie frequency), and in doing so emit discrete forces that have components in three orthogonal directions. These discrete forces are connected in a flux tube and emitted into the external environment. The whole is sometimes termed a particule where it is necessary to differentiate from the point particle of QM. For further details of the internal structures see [26] [29].

One of the implications is that particles are linear structures of finite length they have size. They also have orientation determined by discrete force emission. It is also not relevant to think of the particle as a solid volume of material, or a 


\section{Electron e}

Characterised by one discrete force in each of the three directions. This balanced loading causes the structure to be stable against decay. Physical structure

The discrete forces are released rather than retained as in the photon. Consequently there is an enduring succession of discrete forces in each of the three directions, which creates a long-ranged force effect.

New discrete forces continue to be created and sent down the flux tube at each frequency cycle Inner Fibril provides instantaneous communication between reactive ends, hence a non-local effect Type of reactive end: pulsatile. One reactive end energising and the other de-energising $\left(180^{\circ}\right.$ out of phase)

\section{Notation}

The HED notation represents the distribution of the discrete forces in the three emission directions (HEDs)

Three orthogonal axes $(r, a, t)$ for emission of discrete forces

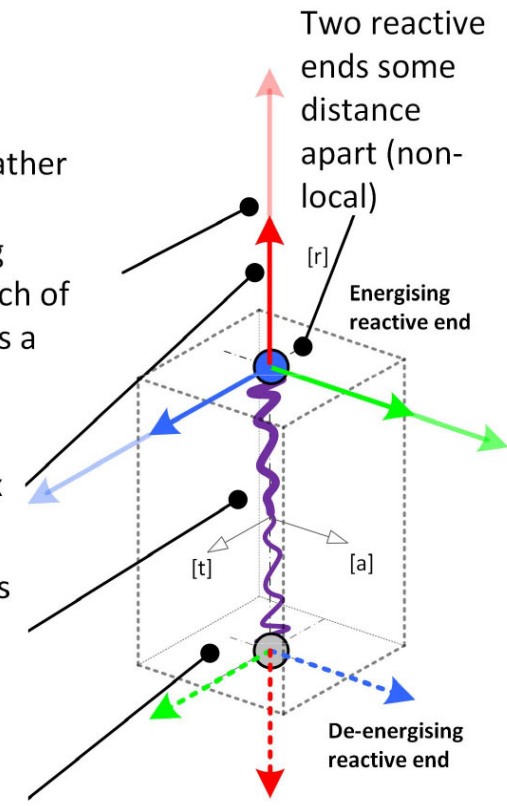

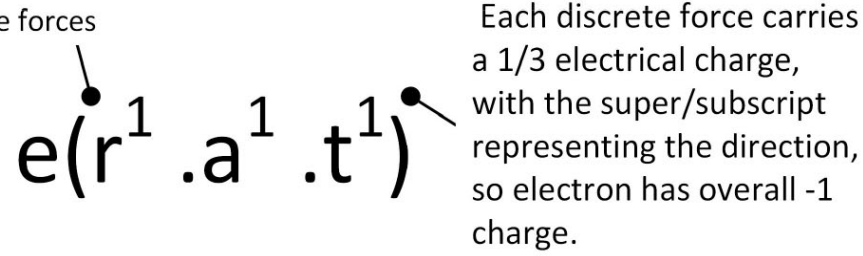

Figure 1. The Cordus design for the electron, showing the reactive ends, fibril, and discrete force emissions. Adapted from [27].

spinning ball of charge. This is especially relevant later when considering the electron spin g-factor.

In this theory, an important difference between the electron and photon is the nature of the emissions. The electron and all massy particles are proposed to emit discrete forces and release them into the external environment to contribute to a fabric of discrete forces. The reactive ends are energized in turn. In contrast the photon is proposed to shunt its discrete force in and out of the fabric, without releasing them. Also both reactive ends are simultaneously active, in opposite directions of transmission. This is important later when considering the Pauli principle. 


\section{Photon y}

Characteristics of the photon are that (1) it does not release its discrete forces, but cycles between emitting and withdrawing them (evanescent), and (2) at any one moment both reactive ends are energised and the discrete forces at both are in the same absolute direction (oscillating).

Discrete force

extended in radial direction

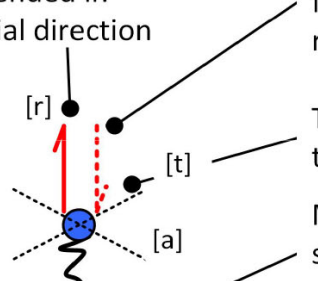

At the next frequency cycle the discrete force is withdrawn from the fabric and reversed

There is no enduring discrete force, so the field effect is local (evanescent)

Motion compensates for incomplete system of discrete forces in the three axes.

Orientation of fibril in space

determines polarisation

Type of reactive end is oscillating: the discrete force is extended then withdrawn, both reactive ends are

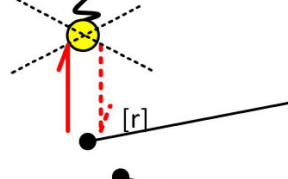
simultaneously active.

Hand system: handParticle interacts at two reactive ends less, as there is no and through its discrete forces. Hence energisation sequence between $[r, a, t]$ axes this is a non-local design.

HED notation

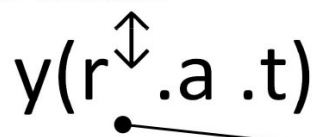

The HED notation is a Cordus symbolic representation of the distribution of the discrete forces in the three emission directions (HEDS)

$\uparrow$ denotes oscillating discrete force, extended and withdrawn

Figure 2. Proposed internal structure of the photon, showing the dual-energization of the reactive ends, and the shunting arrangement of the discrete forces. Adapted from [28].

The theory has been used to explain the following phenomena:

- Wave-particle duality in the double slit device [6], in terms of each reactive end passing through one of the slits.

- Derivation of optical laws from a particle perspective [6], in terms of components of the discrete forces and the effects thereof at reflection and refraction. This includes derivation of critical angle, Snell's law and Brewster's angle.

- Explanation of the decay processes [30] [31] in terms of a contribution by fabric density and loading of neutrino species.

- Prediction of a deeper unified decay model [32] where all the nucleon decay processes (forward, inverse, and induced) may be represented in a single unified decay equation: $\mathrm{p}+2 \mathrm{y}+\mathrm{z}<=>\mathrm{n}+\underline{\mathrm{e}}+\mathrm{v}$ with proton $\mathrm{p}$, photon $\mathrm{y}$, addi- 
tional photons $\mathrm{z}$ where necessary for mass difference, neutron $\mathrm{n}$, electron $\mathrm{e}$, and antimatter species denoted with underscore. Particles, other than photons, change matter-antimatter species when transferred over the equality. This equation may be rearranged to represent $\beta-, \beta+$, and EC in the conventional forward directions, and the induced decays too.

- Explanation for the selective spin characteristics of neutrinos whereby the direction of spin is correlated with the matter-antimatter species [30], in terms of the interaction of the incomplete discrete force structures of these particles with the handedness of the background fabric of discrete forces from other particles.

- Explanation for the annihilation process including a conceptual explanation of the difference between otho- and para-positronium decay rates (ortho and para refer to spin combinations of the bound electron and anti-electron/positron) [33], in terms of the differences in mutual orientations of the two spin states and the effect of the resulting discrete forces on stability.

- Provision of a mechanics for pair production [34] and likewise photon emission [29] [35], in terms of mechanisms that change the particles.

- Structure of atomic nuclei and explanation of stability for nuclides $\mathrm{H}$ to $\mathrm{Ne}$ [36] [37], in terms of a nuclear polymer comprising protons and neutrons in cisphasic and transphasic relationships, neutrons that bridge the polymer, and a predicted morphology of polymer shapes.

- Prediction of a mechanism for asymmetrical baryogenesis in terms of remanufacture of the antielectron (ex pair production) to the proton $8 y+z=>e+$ $\mathrm{p}+2 \mathrm{v}[38]$.

- Explanation of entropy in terms of geometric irreversibility of particles, hence a group property at the bulk level, not a characteristic of the individual particle, which can be reversed at an energy cost at the particle level (Maxwell's agent) [39].

- Nature of the vacuum and the cosmological horizon [40]. In this theory the vacuum comprises a fabric of discrete forces generated by matter particles. The cosmological boundary is the expanding surface where the fabric colonizes the void outside the universe. This theory identifies the infeasibility of placing a physical agent at the boundary of the universe, and also predicts there is no practical way to control the universe from its outer boundary as the holographic principle suggests.

- A theory for time as an emergent property of matter [41], wherein time for a matter particle originates with the frequency cycles of the re-energization of the particle's reactive ends, with relativistic velocity, acceleration, and high gravitation, affecting the energization process. Discrete forces of different particles affect each other via the fabric providing a temporal connectedness of phenomena that are at different geometric locations. The piece-wise communication, via discrete field interactions of the fabric, between adjacent 
volumes of space applies spatial consistency to time, hence space time is discrete.

- Origin of the finite speed of light [42]. On conceptual grounds the density of the fabric determines the electrical and magnetic constants of the vacuum, and gives the speed of light a particular value. A variable speed of light (VSL) is predicted, depending on the fabric density hence the proximity and spatial distribution of matter. Results disfavor the universal applicability of the cosmological principle of homogeneity and isotropy of the universe.

- The time dilation, Lorentz and relativistic Doppler formulations are derivable from the Cordus NLHV particle perspective [43]. The equations contain an unexpected dependency on the fabric density. For a homogenous fabric, which is the assumption of general relativity, the conventional formulations are recovered.

\section{Method}

The purpose of the current work was to prospect for deeper explanations for spin from the Cordus NLHV theory. The present work extends the theory for superposition and entanglement [26].

We used this theory to infer candidate physical structures for spin. We did not find it necessary to change the fundamentals of the theory, though we did identify specific dimensions that were tacit in the original theory. We show that spin may be understood as a geometric attribute of the internal structure of a Cordus particle.

The resulting theory provides a description of spin that is quantized and does not involve orbits. We found that the theory predicts additional spin properties beyond those recognized by QM. The distinction between these properties is lost when one reduces the structure to a point particle, hence the QM perspective is able to be recovered.

We tested the theory for logical congruence against known phenomena of Pauli exclusion, excited states, and selective spin of neutrino species. For the later see also prior work [30]. Explanations were found for these effects, and did not require new assumptions in conflict with the original premises.

We then applied the theory to determine the electron spin g-factor, and quantitatively recovered the Dirac g-factor. We explored the anomalous dipole moment, and found a qualitative explanation for it, but a quantitative derivation was elusive at this time.

\section{Results: A proposed Physical Basis for Spin}

\subsection{Orientation Angles for Spin}

Since Cordus particles have span, they consequently have angular orientation relative to a reference frame. Their frequency behavior means they also have a phase property [44]. We propose these as the basis for spin.

The key spin variables in this theory are the orientation angles of the fibril, 
and the energization phase. If the fibril is orientated with the axis of measurement, then there is only one variable, which is the energization phase, see the electron model above. However in the more general situation the number of dimensions (variables) required to define a Cordus particle is three linear dimensions $[x, y, z]$ for location of a reactive end, one for the length of the span (related to type and energy of the particle), up to three polarisation angles for the orientation of the discrete forces, one composite variable to denote the discrete force content (this differentiates the type of particle) [29], another for the degree of overloading of discrete forces, and one phase variable to indicate which reactive end is energizing. The matter/antimatter hand, which is identified as the hand of energization sequence, requires another variable [33] [45].

Depending on how they are counted, that gives a total of 11 independent variables to fully define a Cordus particle. Not all these dimensions are simple numbers: some like the number and charge of discrete forces are sets, though this is not apparent in the case of the electron but instead becomes evident in say the neutron [31]. The photon has a still simpler discrete force structure and does not need all these variables.

The dimensions of particularly interest for spin are those of orientation. In this theory the spatial orientation of one particle relative to another is defined by several angles: the phase of energization $\theta$, and three orientation angles for the fibril and system of discrete forces $A_{1}, A_{2}, A_{3}$, see Figure 3 and text following.

\subsection{Predicted Sub-Types of Spin}

We identify several different types of spin within the Cordus theory. It is proposed that not all these are manifest in every situation, and some are predicted only to be evident at a finer scale. Several of these spin variables are naturally inaccessible to representation in quantum theory because it assumes particles are 0 -D points.

\section{Number of Reactive ends (E)}

The number of reactive ends in the particle, which is two rather than say three, indicates the energization frequency model of the particle. For the Cordus theory $\mathrm{E}=2$ for a single particle. For $\mathrm{QM}$, and any theory built on a 0 -D point construct, the number of reactive ends is $\mathrm{E}=1$. For electromagnetic wave theory, where a dipole construct is sometimes used, $\mathrm{E}=2$.

Intra-Energisation state $(s)$

This indicates the energization state of the reactive end at the moment under examination. The $s$ variable denotes the energization state of a reactive end at the moment in question. For matter particles like the electron, one reactive end is energizing $\mathrm{s}(1)$ while the other is de-energizing $\mathrm{s}(0)$, i.e. the two reactive ends are $180^{\circ}$ out of phase, hence an $s(1,0)$ structure. The reactive ends thus pulse with discrete force emissions. There is no emission of discrete forces at the de-energized state. At each $1 / 2$ frequency cycle the state of any one reactive end changes. 


\section{Cordus orientation variables}

Free electron relative to an arbitrary frame of reference $[x, y, z]$

Phase angle of re-energisation, $\theta$

(a) For multiple particules in a decoherent relationship, this is a continuous variable.

(b) For particules in a coherent assembly, i.e. bonded by the synchronous interaction, it is a discrete variable of $\theta=0$ or $\pi$

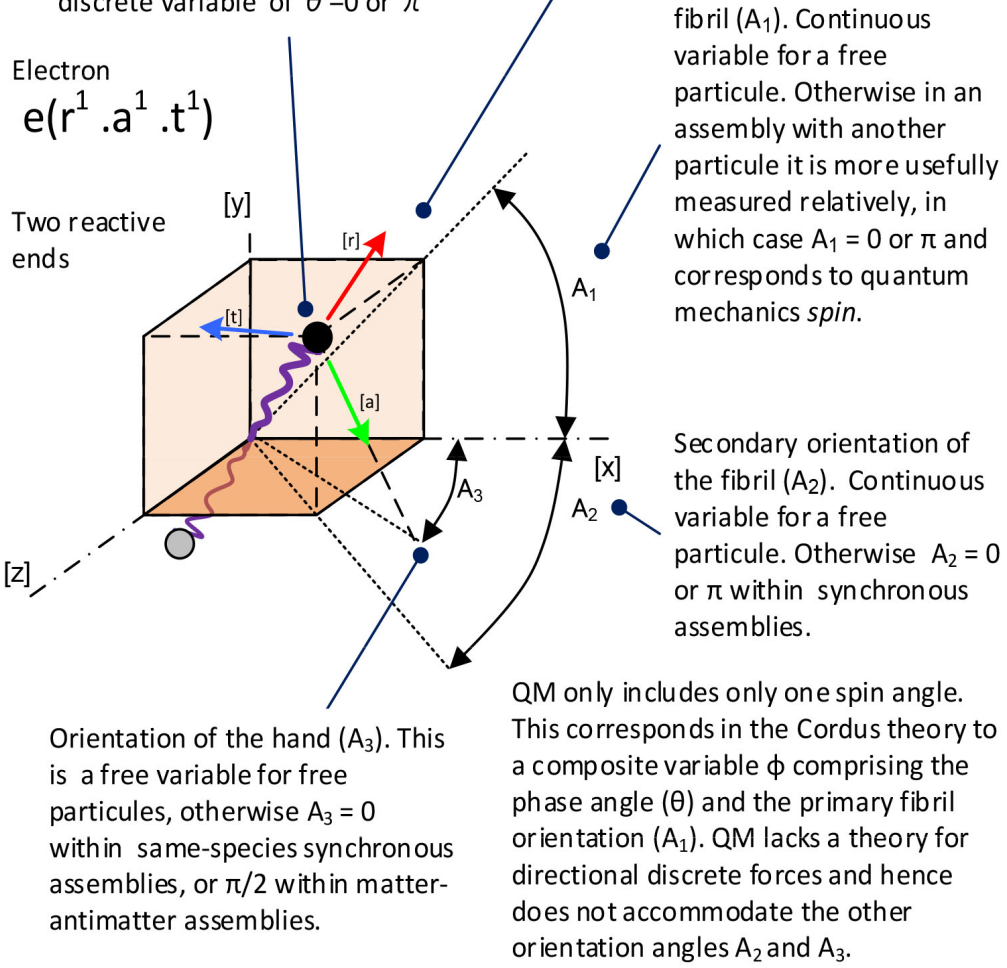

Figure 3. Definition of Cordus orientation variables that define spin.

For the photon, both reactive ends are simultaneously active. At any one moment, one reactive end is emitting a discrete force and the other is retracting its emission, $s(+1 / 2,-1 / 2)$. The photon oscillates its emissions. The reactive ends are simultaneously active, though in different directions. At the next $1 / 2$ frequency cycle the state of the reactive ends changes.

Nuclides with even numbers of nucleons and symmetrical polymers emit discrete forces simultaneously in all directions, though from different locations in the polymer [36]. At a sufficient coarse scale of observation, where the span of the polymer is considered to be zero, this would appear to be a particle with simultaneous emissions in all directions, i.e. $\mathrm{s}(1,1)$. This, we propose, is the basis of the bosonic attribute of nuclides with even numbers of nucleons.

Fibril orientation angle $\left(A_{1-3}\right)$

This measure of spin refers to the orientation of the fibril of a single particle, relative to another particle or frame of reference. The necessary parameters are 
two angles $A_{1}$ and $A_{2}$ describing the orientation of the span, and a third angle $A_{3}$ for the alignment of the [a] axis. These apply to massy particles and the photon. They correspond to polarisation angles for the photon in electromagnetic wave theory.

Inter Phase angle $(\theta)$ - cis and transphasic

There is a relative phase angle $\theta$ of energisation between two neighboring particles. If the particles are in a coherent relationship, which requires synchronization of discrete forces and a common frequency of the energization $\omega$, then the only options are $\theta=0$ (cisphasic) or $\theta=\pi$ (transphasic) energization [33] [44]. In the more general case of a discoherent relationship then there are no restrictions on $\theta$. In this way the Cordus theory provides a means to differentiation coherent and discoherent (discord) states of matter. This is difficult to explain with other theories.

Discrete force pairs

The difference in orientation of matter-antimatter discrete force pairs is interpreted as a form of spin at a deeper level within the particle. The Cordus notation for these is $x_{1}^{1}$ and $x_{1}^{1}$ [30] [33]. This concept is not needed for the present discussion, but is included for completeness as it is important in the proposed Cordus processes for decay and the weak interaction [31], photon emission [35] and pair production [34]. It is also relevant in the solution for asymmetrical baryogenesis [38].

Angular momentum ( $M)$

This spin refers to angular momentum. The interpretation is of a free Cordus particle rotating about an axis. For an individual particle or decoherent assemblies of particles this spin may be a continuous value. However in coherent systems it is quantized due to the synchronicity of the interactions between the particles [44]. Hence it is proposed that the quantized nature of spin arises from the coherent assembly of particles, specifically from their mutual alignment of emission directions.

Handed motion $(H)$

Spin hand refers to the direction of the angular momentum relative to the direction of motion, and may be clockwise or anticlockwise. This is a geometrically simple concept but it has potentially profound implications because it explains the selective spin characteristics of the neutrino matter-antimatter species, see below and [30]. Hence it is possible with this theory to explain why neutrinos spin the one way, and anti-neutrinos the other. This has otherwise not been explained with conventional QM.

Having proposed the origins of spin variables at the sub-particle level, we next apply these principles to explain several phenomena.

\section{Applications of Cordus Theory to Quantum Phenomena}

\subsection{Empirical Measurement of Spin}

The Cordus theory proposes that measured spin corresponds to phase and an- 
gular orientation of the fibril of the particle.

This is consistent with how spin is measured empirically. In a coherent light source the photons are produced with a certain orientation, and this occurs either at emission or by subsequent filtering using polarizers to exclude non-compliant orientations. Also the component of electric field, hence component of spin, may be measured in an axis set by the observer. These light sources produce many photons and the probabilities measured by quantum mechanics represent these components and the underlying stochastic variability. A number of photons are sacrificed for measurement purposes, and used to infer the properties of the wider ensemble. Hence also, a decoherent light source produces photons with uncontrolled orientations, and this is represented in quantum mechanics as indeterminate spin. The Cordus theory is consistent with these results, but explains them as arising from the geometric properties of the particle. Thus it is proposed that the aligned molecules within polarising filters really do selectively obstruct photons that have orientation that is non-compliant with the filter.

\subsection{Coherence and Decoherence}

The Cordus theory proposes that coherence arises when adjacent particles synchronize the phase of emission of their discrete forces.

Within the Cordus explanation for spin there is a differentiation between coherent and decoherent assemblies of particles [44]. This is not a distinction explainable with quantum theory. The Cordus theory proposes that the formation of coherence between two or more matter particles requires their acceptance of a common (or harmonic) frequency, and a common phase of emissions. This is because the bonding is via the synchronous interaction (strong force), which as the name implies requires synchronous emission of discrete forces [44]. This fixes the frequency $\omega$ of the particle to a common or harmonic value.

The synchronous interaction also makes the three orientation angles $A_{1}, A_{2}$ and $A_{3}$ into local constants, so they are no longer variables. The only remaining variable is the phase angle $\theta$, which in the coherent case is either cisphasic $(\theta=$ 0 ) or transphasic $(\theta=\pi)$. Consequently for coherent assemblies of matter, both frequency and phase are no longer variables for an individual particle but are instead group properties. We propose this as the physical mechanism underpinning superfluidity, superconductivity, and Bose-Einstein condensates (see below).

Multiple particles that are in decoherent assembly have their own independent parameters for all these variables. Such assemblies are predicted to interact via the electro-magneto-gravitational (EMG) forces instead of the synchronous force.

As this shows, physically meaningful definitions of spin are provided in this NLHV design. However there are more spin variables here than provided in quantum theory. This can be explained as follows. QM assumes that all particles are in a coherent assembly state, which means that all the angles of polarisation 
are fixed, and the frequency too. Consequently the only spin variable left in a coherent body is the phase $\theta$, which can only take two values (since the particle has two reactive ends). This explains why spin is discrete in quantum mechanics. QM does not extend to describe ensembles of decoherent particles, which is what the other Cordus variables are used for.

\subsection{Pauli Exclusion Principle}

The Cordus theory proposes that pairs of electrons can share a common space by arranging to have transphasic (opposite phase) inter-particle relationships.

This theory may be applied to understand the interaction between electron Pauli pairs in orbitals. The two electrons in an orbital are known to have opposite spin when measured, hence the Einstein-Podolsky-Rosen paradox. This is considered a paradox because it is unclear how the two particles interacted to communicate their states to each other to contrive such a result.

The Cordus theory explains the situation as follows. The two electrons share locations for reactive ends but in opposite (transphasic) re-energisation phase, see Figure 4. This transphasic interaction protects the emission directions of the assembly, and is thus energetically favourable for the individual participants. This also explains why there are only two electrons in each orbital, not more, hence the Pauli Exclusion Principle. Hence the two electrons are always found to be in complementary states when measured. The fact that the electrons are sharing the orbital means that they have pre-arranged with each other (and the nucleus) to be in this complementary state even before the Observer started the interrogation, so to the Observer the outcome of the experiment looks like an act of contrivance by the particles. However that is merely an artefact of observation.

Note that it is not the absolute orientation of the particle that is proposed to be important, but the relative orientation between the two particles. In a coherent system, the two particles can only be either in phase with each other or out of phase, hence only two spin states are possible for Pauli pairs. In the more general case where two electrons are not in coherence with each other, there are infinitely many orientations that the fibril may take. This is proposed as the reason why the Pauli exclusion principle only applies in special situations like orbitals.

In this context the Cordus particle concept can also be extended to larger assemblies such as atomic nuclei [46]. It is able to explain why each of the nuclides $\mathrm{H}$ to $\mathrm{Ne}$ are stable, unstable, or non-existent [37]. It does this by proposing that the protons and neutrons are also linear structure like the electron, and that these form closed chains or nuclear polymers [36]. The polymers are stabilised by bridge neutrons, which accommodates the empirical observation that heavier elements need more neutrons for stability.

\subsection{Excited States and Photon Emission}

The Cordus theory proposes that excited states comprise one electron in a set adopting a higher harmonic frequency, while still retaining synchronous interactions with the basal particle(s). 


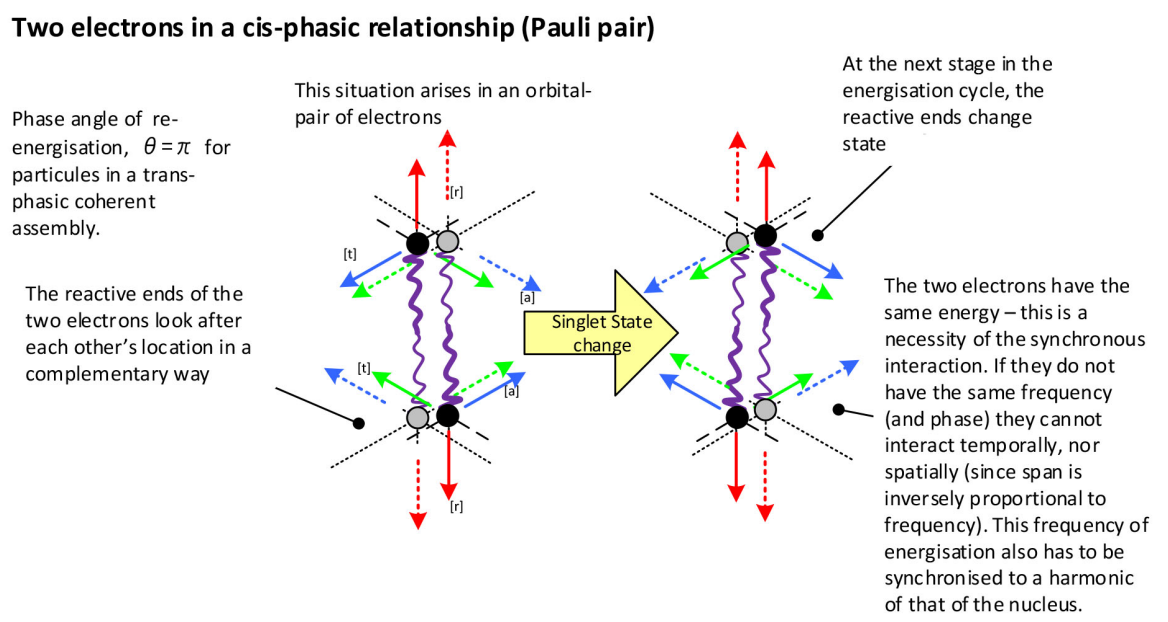

Figure 4. The Cordus explanation for the spin arrangements for a Pauli electron pair.

The behaviour of excited states can also be understood in terms of this Cordus theory. In an excited state one of the electrons (B) in a Pauli pair absorbs energy. In the Cordus explanation, this energy causes the B electron to increase its frequency and decrease its span. It therefore moves into partial temporal and spatial de-synchronisation with electron A (which remains in the ground state). B can persist in this state by finding a harmonic frequency with which to interact with $\mathrm{A}$, a type of spin gearing. However the interaction is also, via $\mathrm{A}$, with the rest of the nucleus. The nucleus has a large resistance to changing its spin attributes, due to its large mass.

Electron B may transfer some energy into electron A and the nucleus, as part of the process of negotiating a mutually acceptable set of harmonic frequencies. Or it may emit the energy as a photon. Emission is also covered by this theory [29] [35]. This is a precise interaction and any excess energy above that needed for the relevant frequencies is a hindrance, and is discarded. Thus the orbital system takes only certain discrete energy states. Hence the quantum nature of discrete energy levels can also be qualitatively explained using this NLHV theory. This negotiation process takes frequency cycles, hence time, and thus the transitions are not instantaneous.

The relationship between electrons A and B thereby changes from the direct 1:1 synchronicity of the $\uparrow \downarrow$ state (this notation refers to the discrete forces, see Figure 1), to one where electron $B$ is at a higher harmonic frequency. $B$ is then trapped in this state. Subsequently an external perturbation means that $B$ is no longer in an energetically sustainable position. It is expected that B will more easily revert back to the ground state when it and A are momentarily in a cisphasic relationship $\uparrow \uparrow$ (or $\downarrow \downarrow)$ ). For it to make the transition back to the transphasic state $\uparrow \downarrow$ it needs to change its frequency to that of A, and move into the opposite phase. The Cordus theory separately identifies mechanisms whereby a particle can skip half a frequency cycle by emitting a photon [29]. If this photon also carries away the energy difference, then the spin adjustment is 
complete, and the intersystem crossing occurs. Hence this is a phosphorescence emission.

\subsection{Selective Spin of Neutrino Species}

This theory proposes that the physical mechanism for the matter-antimatter species differentiation is the handedness of the energisation sequence of the discrete forces [45]. Given three axes, there are only two such sequences, which are dexter and sinister (right and left). Expansion of this concept has been used to explain annihilation [33], pair production [34], and asymmetrical baryogenesis [38]. When coupled with the concept of spin described above, the theory also explains the unique spin characteristics of the neutrino species [30]. The physical evidence is that the neutrino always spins one way relative to its motion (left handed), and the antineutrino the other (right handed).

The proposed mechanism is that the neutrino species have incomplete discrete force emission and hence must recruit discrete forces from the fabric. This results in reactive translational and rotary motions. The direction of spin motion is determined by the energisation sequence, and this is also the matter-antimatter species differentiation, see Figure 5 and Figure 6.

Up to here the explanations for spin phenomena have been quantitative. We now demonstrate that the theory quantitatively recovers the principle component of the electron g-factor.

\section{Electron Spin G-Factor}

Thompson's plum-pudding model of the atom proposed electrons in a matrix of positive charge, making up a solid ball. That concept of solidity was disproved by Rutherford [47] in the gold-foil experiments. This led to the modern idea of quantum mechanics, with a nucleus and electrons in orbitals. Even though quantum mechanics treats particles as $0 \mathrm{D}$ points, there is still an acceptance that particles occupy space stochastically such that macroscopic matter has volume. For example the proton has an empirically known charge radius. It is invariably assumed that such particles occupy the whole of their volume, even if non-uniformly and not continuously in time. This assumption is important in what follows.

Dirac explored the assumption that the electron had its charge on an outer conductive spherical surface [48]. If particles like the electron were solid spheres, or comprised sub-particles in a solid spherical matrix, and if the same sub-particles contributed fractionally to both charge and mass, then it would be expected that the moments of charge and mass would be the same.

Empirical evidence shows this not to be the case, and suggests that the internal sub-charges would need to be distributed differently to the sub-masses. More specifically, the $g$-factor represents the constant of proportionality between the magnetic dipole moment $\mu_{\mathrm{s}}$ which measures spin of a charge, relative to the spin angular momentum $\mathrm{S}$ which measures the moment distribution of mass. The 


\section{Neutrino $\mathbf{v}$}

The neutrino is neutral since it has equal positive and negative charged discrete forces. the arrangement of those discrete forces is different to that of the neutron.

The HED energisation sequence is expected to create a corresponding spin angular momentum the direction of which depends on the hand. Hence left-spin-hand arises from dexter hand energisation sequence.

Motion arises in the [at] plane as the particule lacks its own discrete forces in these axes. Where the fabric exists it uses discrete forces from the fabric, and then propagates at the speed of light as that is the saturated speed of the medium.
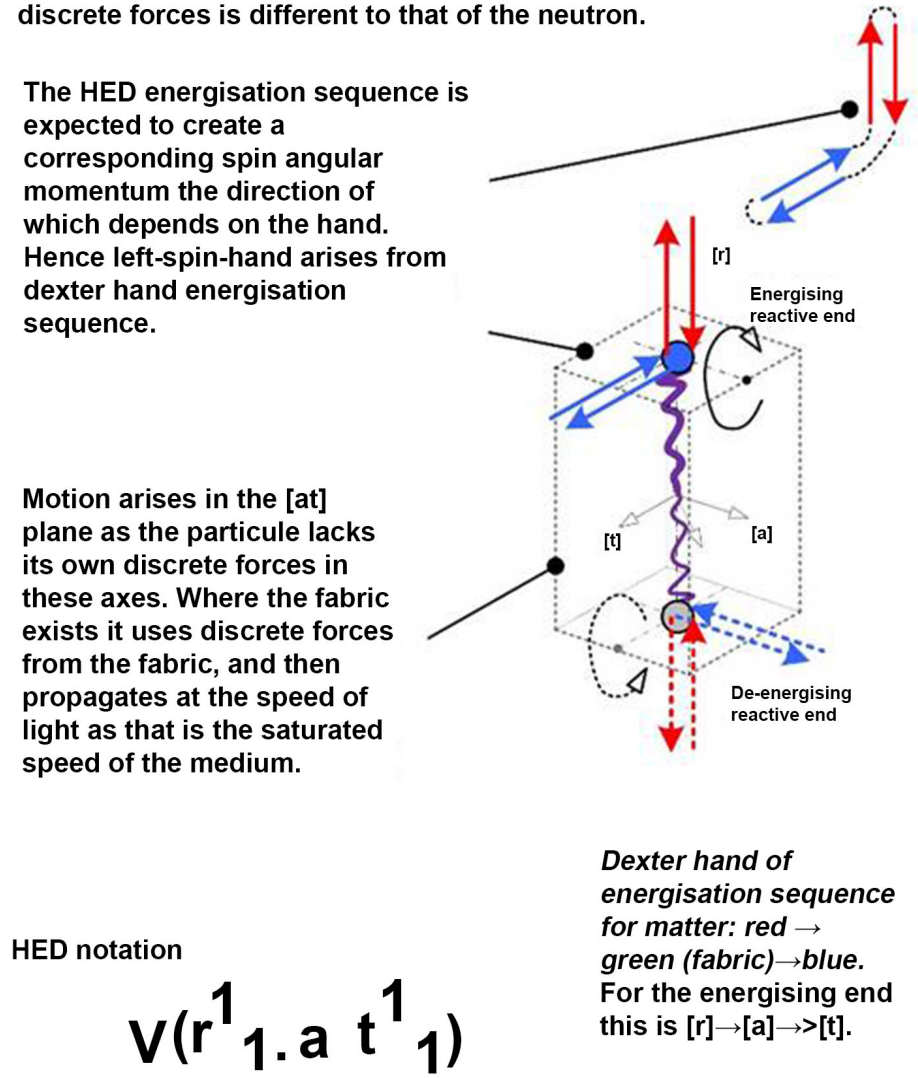

Layout is representative: Anticipate that discrete fields may dynamically relocate to other HEDs as the span changes orientation due to linear and rotary motion.

The HED notation is a Cordus symbolic representation of the distribution of the discrete forces in the three emission directions (HEDs)

Figure 5. Proposed structure of the neutrino, including internal structure and external discrete force structure. The imbalance of the discrete forces results in linear and spin motions. The latter is uniquely determined by the energisation sequence, hence matter-antimatter species. Image adapted with permission from [30].

Dirac electron spin g-factor is approximate twice the spin, more accurately 2.00231930436153. That this is about 2 rather than 1 is evidence that the charge of the particle is distributed very differently to its mass. This is considered one of the key characteristics of QM, since no other theory of physics has been able to explain why $g=2$. There is a further triumph for $\mathrm{QM}$, since the anomalous magnetic dipole moment (the discrepancy from 2) can be calculated to high accuracy by quantum electrodynamics [49].

\section{Explanation of electron g-factor with Cordus particle theory}

In what follows we show that the new theory is able to derive $g=2$. In the Cordus theory the electrostatic field strength of an electric charge is determined 


\section{Antineutrino $\underline{\mathbf{v}}$}

The antineutrino has the same discrete force count as the neutrino, but the opposite hand of energisation sequence The unique spin directions of the neutrino and antineutrino arise due to the hand differences.

The timing of the HED energisationwhich is sinisterhand-creates a spin angular momentum.

Incomplete HED a activation creates a reaction torque against the fabric, hence a spin, the direction of which depends on the hand (energisation sequence), hence $\underline{v}$ always has right-spin hand.

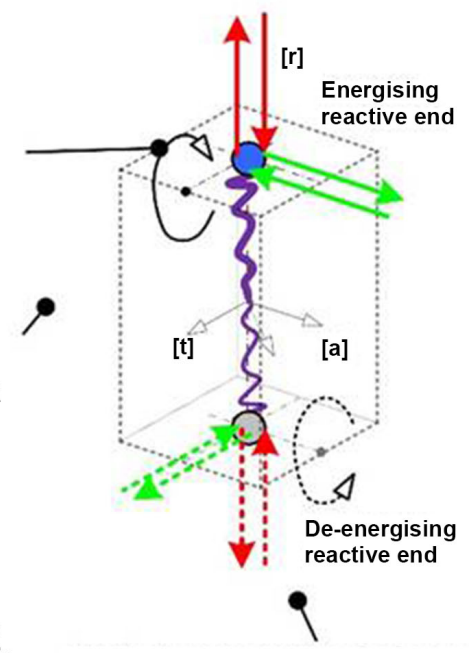

Motion arises in the lat] plane as the particule lacks its own discrete forces in these axes. Where the fabric exists it uses discrete forces from the fabric, and then propagates at the speed of light as that is

HED notation the saturated speed of the medium.

$$
\underline{v}\left(r_{1} \cdot a \cdot \underline{1}_{1}{ }^{1}\right)
$$

It is anticipated that discrete forces may dynamically relocate to other HEDs, perhaps in a cyclic manner, as the span changes oriebtation relative to the linear and rotary motions.
Sinister hand of energisation sequence for matter: red $\rightarrow$ blue ( fabric) $\rightarrow$ green. For the energising end this is $[r] \rightarrow[t] \rightarrow[a]$.

Figure 6. Proposed structure of the antineutrino, including internal structure and external discrete force structure. This particle spins in the opposite direction to the neutrino, because of the difference in energization sequence of the discrete forces. Image adapted with permission from [30].

by the signed sum of discrete forces emitted by that charge. The theory predicts, in contrast, that mass is determined by the total number of discrete forces, irrespective of their charge. Hence some particles (e.g. the neutron) emit charge-neutral pairs of discrete forces that contribute to mass but not to charge [31]. This is an important difference. In neutral particles, such as the neutron, it is proposed that both positive and negative discrete forces are emitted so the net electrostatic field strength is zero, but there are still discrete forces contributing to the mass and gravitational effect. In other particles, like the proton, both effects exist: there is a net external charge and a neutral part. These are the overt and implicit (or covert) parts respectively [32]. Thus the implicit discrete forces 
contribute to mass, and hence to spin angular momentum. In this theory, mass is also determined by the energisation frequency, whereas charge is not. Hence higher frequencies cause more discrete forces to be emitted in unit time, hence greater mass.

Hence the Cordus theory predicts different mechanisms for the electric field and mass. In contrast the classical perspective is of a spherical solid body with a radial dispersion of both charge and gravitational field. For the electron, in the Cordus theory, the discrete forces are identified as a complete set of one emission in each of the three axes, hence $e=\left[\mathrm{r}^{1}, \mathrm{a}^{1}, \mathrm{t}^{1}\right]$ without covert discrete forces [34]. This sums to one unit of charge and one unit of mass. We show that the electron $\mathrm{g}$-factor for this arrangement is 2 .

Start by noting that the electron spin g-factor is a constant included in the Dirac particle equation:

$$
\mu_{e}=g_{e} \cdot \frac{\mu_{B}}{\bar{h}} S_{e}
$$

where $\mu_{e}$ is the electron magnetic moment which measures the distribution of charge, $g_{e}$ is the electron spin g-factor, $\mu_{B}$ is the Bohr magneton, $e$ is the electron charge, $\bar{h}$ is the reduced Planck constant, and $S_{e}$ is the spin angular momentum which measures the distribution of mass.

Identify the Bohr magneton, where $m_{e}$ is the electron mass:

$$
\mu_{B}=\frac{e \bar{h}}{2 m_{e}}
$$

Hence by substitution and rearrangement:

$$
g_{e}=2 \frac{\mu_{e} / e}{S_{e} / m_{e}}
$$

The term $\mu_{e} / e$ is the moment of charge per unit charge, and $S_{e} / m_{e}$ is the moment of mass per unit of mass.

In the Cordus theory the mass and charge interactions occur at the reactive ends, since the discrete forces provide the underlying mechanisms of causality. Hence it is at the ends of the span that the discrete forces act. Furthermore, the frequency of emission for the charge and the mass is the same, since both are serviced by the underlying energisation process: the electrostatic force is proposed to be from the linear action of the discrete forces, and the mass \& gravitation from the torsional action of the same complex of discrete forces. Both effects originate at the reactive ends. Thus the Cordus theory predicts that the moment arm for charge is the same as that for mass, hence:

$$
\mu_{e} / e=S_{e} / m_{e}
$$

The above moment arm considerations are important. In contrast the classical perspective is that the mass is contained uniformly inside a spherical volume whereas the charge is distributed on the surface of that volume, hence different moment arms for the two effects.

Substituting Eqnation (5) into Eqnation (4) gives the electron spin g-factor 
per the Cordus NLHV theory:

$$
g_{e}=2
$$

This recovers the Dirac electron spin g-factor. This finding disconfirms the classical idea of a particle being a simple spherical solid body. The finding is consistent with QM but independent thereof and derived from a NLHV basis.

This is novel as the derivation is from a NLHV particle theory. Previously the only theory of physics to explain this result has been quantum theory. Providing a derivation using the Cordus particle theory shows that the phenomenon is not a uniquely quantum effect. We have shown this may be accomplished assuming a particle structure with two reactive ends, in contrast to classical mechanics that assumes a spherical particle, and QM a 0-D point particle. Note that this Cordus particle structure was originally derived for a different phenomenon [6], rather than being specifically designed to explain spin, and hence there is coherence across the range of phenomena explained by the theory.

\section{Anomalous magnetic dipole moment}

The empirical evidence is that $g_{e}$ is slightly more than 2 , i.e. that the moment of charge is larger than the moment of mass, $g=2.00231930436153$. This small difference is called the anomalous magnetic dipole moment. It is explained by quantum electrodynamics as an interaction between the electron and one or more virtual photons. QED is able to calculate $\mathrm{g}_{\mathrm{e}}$ to high precision, which is one of the great successes of the standard model.

The Cordus theory explains the anomalous magnetic dipole moment as an interaction between the electron and the fabric. The fabric in this theory comprises the volume of space containing the discrete forces emitted by all the particles in the observable universe [40] [42]. The energisation of a reactive end creates and emits a new discrete force into this fabric. There will exist random events when a discrete force in the fabric happens to be co-located and aligned (cis) or anti-aligned (trans) with this new emission, and the response of the particle is slightly asymmetrical.

Where the discrete forces of the electron and the fabric are aligned the effect is to momentarily retard the emission of the discrete force, i.e. postpone the effect of the charge into the future. This fractionally reduces the strength of the charge in the present moment. For the case where the discrete forces are anti-aligned, the combination creates the structure of a photon [29]. This new photon has a brief existence in the fabric. The brevity is due to the two discrete forces, one from the fabric and the other from the electron, being members of separate flux tubes with spatially diverging commitments. Consequently the created photon is merely a temporary random alignment. This is similar to the QM concept of 'virtual photon'. The temporary photon moves a small distance in its brief existence, and then separates into its constituent discrete forces. However we propose that it can occasionally be absorbed by a third particle, such as a passing neutrino. Hence the anti-aligned pairing fractionally neutralises charge, hence reduces effective charge while preserving charge moment. The effect is less pro- 
nounced for mass, because of the greater statistical improbability of three discrete forces from the fabric having the necessary coincidence. Inspection of Eqnation (4) shows that the effect of these two asymmetries is to increase the $g$ factor above 2. This is not inconsistent with the Schwinger radiative correction [49].

Likewise other fortuitous alignments of discrete forces may mimic the discrete force structure of other particles, such as electron-antielectron, or quark-antiquark pairs, and thereby create virtual particles of these types too. These will also make a small contribution to fractionally decreasing the effect of charge. However these other particles have more complex discrete force structures, and hence the probability of these structures being correctly presented by the randomness in the fabric are smaller. Hence heavier virtual particles will be rarer and make a smaller overall contribution. This is similar to the QED prediction of a secondary contribution by hadronic vacuum polarisation [50].

\section{Muon g-factor}

The $\mathrm{g}$ factor effect is not proportional to mass, within a family of particles, because the spin angular momentum scales proportionally with increased mass, e.g. for the muon $S_{\mu} \propto m_{\mu}$. Nonetheless the muon $g$ factor is not identical to that of the electron, but is instead slightly greater with $g_{\mu}=2.0023318414$. Our explanation is that the higher energisation frequency of the muon causes it to emit discrete forces more often, and hence a greater exposure to forming a virtual photon with a discrete force from the fabric. These interactions decrease the effective charge and increase the $\mathrm{g}$ factor. In contrast the standard model imputes this to greater access to heavier virtual particles.

The implication is that the fabric density affects the production of virtual photons. The Cordus theory predicts that the production of virtual photons will be proportional to the fabric density, and the alignment thereof with the particle. We make the falsifiable prediction that the anomalous magnetic dipole moment is not universally constant. Instead we predict $g_{e}$ will be greater in situations of higher fabric density (e.g. regions of higher gravitational field strength or denser galaxies or relativistic velocities), and should display a correlation with orientation (e.g. spin relative to alignment towards charged objects or large bodies of coherent matter). Since the fabric density is temporally and spatially variable in the universe, this further implies that the anomalous magnetic dipole moment changes with epoch and location in the universe. An interesting future research question is whether the anomalous moment might be used to determine the absolute value of the local fabric density.

It makes sense that the production process of virtual photons should depend on the fine structure constant $\alpha$. This is because $\alpha$ is interpreted in this theory as 'a measure of the transmission efficacy of the fabric, i.e. it determines the relationship between the electric constant of the vacuum fabric, and the speed of propagation $c$ through the fabric' [29]. Hence $\alpha$ relates to electrical forces and propagation of fields, which includes electron bonding. 


\section{Discussion}

\subsection{Commentary}

Starting from first principles of geometry, we have shown that physical structures at the sub-particle level can explain multiple spin phenomena including polarisation, features of coherent-decoherent assemblies, Pauli exclusion principle (Einstein-Podolsky-Rosen paradox), excited states, and selective spin of neutrino species. We finished by recovering the electron spin $\mathrm{g}$-factor $\mathrm{g} \approx 2$, and explaining why the anomalous magnetic dipole moment and muon g-factor are greater.

\subsection{Implications}

We have shown that phenomena considered to be uniquely quantum may be explained by theories other than QM. The conventional interpretation is that the electron g-factor precludes the possibility of fundamental particles having internal structure. Hence QM asserts that spin truly is an intrinsic property. The present work falsifies this by deriving the g-factor using NLHV structures without recourse to quantum theory.

We have achieved this by departing from the conventional assumption that any hidden variable solution would comprise smaller particles rotating about a central mass, somewhat like planets orbiting the sun, the defunct plum-pudding model, or the extant bag models of nuclear structure. Such designs would indeed not explain the g-factor. However there is no need to limit the design of a NLHV solution to an orbital arrangement. By conceptualising a radically different arrangement, we have shown that the g-factor may be recovered.

The g-factor result is more than an interesting curiosity, because it does not stand alone. The same theory has been applied to many other phenomena. It derives from first principles the laws of optical reflection and refraction [6], explains the stability of the atomic nucleus [36] [37], derives the Lorentz factor [43], explains asymmetrical baryogenesis [38]. Other parts of the work address time [41], entropy [39], and the strong force (synchronous interaction) [44]. The theory is logically consistent across all these explanations. This strengthens the case for a new physics based on hidden variables.

The wider implication is that the next deeper level of physics would be based on particles having sub-structures. While the possibility of a non-point structure has been considered from the outset of quantum theory [51], up to now the difficulty has being devising an alternative structure. This is especially as the Bell type inequality tests precluded many types of internal structure [17]. However the specific structure predicted by the Cordus theory is not precluded by the Bell tests [26]. The new theory implies that there is a deeper determinism to particle behaviour, which is approximated by the stochastic representation of quantum mechanics. As shown in the above references, a unification between features of particle behaviour and general relativity is conceptually feasible at this deeper level. This is another attractive feature of the theory. 


\subsection{Limitations}

The limitation of the theory is the lack of a mathematical formalism. In this regard quantum mechanics is much superior. We derived the basic form of the electron g-factor using a mathematical approach, but not the anomalous part. We have yet to find a form of mathematics to represent the Cordus theory - this is an open problem. The number of geometric variables in the Cordus particle is broadly consistent with string/ $\mathrm{M}$ theory, though the theories come at the problem with different approaches. Possibly this hints at a correspondence, in which case some of the string theories might be formulated to create a mathematical representation of the Cordus particle structure.

\subsection{Implications for Future Research}

We have only addressed the first of the questions identified at the outset: how does spin arise at the fundamental level? The other questions remain: Why are nuclides with odd total of nucleons fermions, while those with even totals are bosons? Why do some bosons (photons) stack, whereas other bosons like $2 \mathrm{He} 2$ nuclei do not co-locate? Why only $1 / 2$ spin for elementary fermions and predominately 1 for bosons? What is the physical mechanism for the Einstein-de Haas and Barnett effects?

\section{Conclusions}

This work makes several original conceptual contributions. We propose that the spin property arises from the internal structure of particles, and this is new. We have predicted what those structures are, and how they relate to spin. Consequently, the work provides a physical explanation for spin, which has not been achieved before.

The new spin theory provides a conceptual explanation for a variety of observed spin behaviors. Existing quantum based theories already provide quantitative formalisms in some cases, but an ontological explanation has been lacking.

Another contribution is the advancement of the non-local hidden-variable branch of physics. By addressing the spin behaviors and deriving the electron $\mathrm{g}$-factor, the comprehensiveness of the Cordus theory has been enlarged. The theory provides a single coherent framework that explains spin (this paper), photon absorption \& emission [29] [35], matter/antimatter \& annihilation [33] [45], nuclide structures and stability (to at least $\mathrm{Ne}$ ) [36] [37], strong interaction [44], weak interaction [31], decay sequences [30] [32] [52], asymmetrical genesis (baryogenesis and leptogenesis) [34] [38], time dilation [41] [43], aspects of cosmology [40] [42], entropy [39], and wave-particle duality [6] [26]. This is original as it provides wider coverage than other NLHV theories.

This theory explains multiple spin phenomena that are held to be uniquely quantum effects: Pauli electron pairs, excited states, and the electron spin g-factor $g \approx 2$. An explanation, albeit qualitative, is also offered for the anomalous component. Explaining these using a theory of physics other than QM is 
original.

Another accomplishment is offering an explanation of the selective spin characteristics of the neutrino species. This has not been explained with other theories.

In summary the work demonstrates that a physical basis can be conceived for spin, and that the electron g-factor can be explained by NLHV theory. Consequently, we reject as unnecessary simplification the QM premise that particles are $0 \mathrm{D}$ points and particle properties merely intrinsic, and instead we propose the principle of physical realism applies. We suggest the idea that particles do have internal structure is a promising concept for advancing fundamental physics.

\section{Acknowledgements}

Parts of this paper are based on an earlier unpublished work [53]. We acknowledge the contribution of Ariel Pons to those early ideas.

\section{Contribution Statement}

All authors contributed to the general development of the theory. DP led the development of the specific explanations provided here. DP wrote the first draft of the paper and all authors contributed to its finalisation.

\section{Conflicts of Interest}

The authors declare no conflicts of interest regarding the publication of this paper.

\section{References}

[1] Xiang, S.-H., Deng, X.-P., Song, K.-H., Wen, W. and Shi, Z.-G. (2011) Physica Scripta, 84, Article ID: 065010. https://doi.org/10.1088/0031-8949/84/06/065010

[2] Julsgaard, B., Kozhekin, A. and Polzik, E.S. (2001) Nature, 413, 400-403. https://doi.org/10.1038/35096524

[3] Hasegawa, Y., Loidl, R., Baron, M., Badurek, G. and Rauch, H. (2006) Physica B: Condensed Matter, 385-386, 1377-1380. https://doi.org/10.1016/j.physb.2006.05.208

[4] Chen, W., Shen, R., Sheng, L., Wang, B.G. and Xing, D.Y. (2012) Physical Review Letters, 109, Article ID: 036802. https://doi.org/10.1103/PhysRevLett.109.036802

[5] Dirac, P.A.M. (1942) Royal Society of London-Proceeding, 180, 1-40. https://doi.org/10.1098/rspa.1942.0023

[6] Pons, D.J., Pons, A.D., Pons, A.M. and Pons, A.J. (2012) Physics Essays, 25, 132-140. https://doi.org/10.4006/0836-1398-25.1.132

[7] Hasegawa, Y., Loidl, R., Badurek, G., Baron, M. and Rauch, H. (2003) Nature, 425, 45-48. https://doi.org/10.1038/nature01881

[8] Stone, N.J. (2014) Table of Nuclear Magnetic Dipole and Electric Quadrupole Moments. International Atomic Energy Agency, Wien, INDC(NDS)-0658. http://www.iaea.org/inis/collection/NCLCollectionStore/ Public/45/029/45029196. 
pdf

[9] Samsonenko, N.V. and Katchat Chkhotu, L. (1986) Soviet Physics Journal, 29, 562-565. https://doi.org/10.1007/BF00895504

[10] Saglam, M. and Sahin, G. (2009) International Journal of Modern Physics B, 23, 4977-4985. https://doi.org/10.1142/S0217979209053862

[11] Pei, Y.-J. (1996) Zeitschrift für Physik C: Particles and Fields, 72, 39-46. https://doi.org/10.1007/s002880050221

[12] Lyuboshitz, V. (2009) Physics of Atomic Nuclei, 72, 311-318. https://doi.org/10.1134/S1063778809020173

[13] Barger, V., Chiang, C.-W., Keung, W.-Y. and Marfatia, D. (2011) Physical Review Letters, 106, Article ID: 153001. https://doi.org/10.1103/PhysRevLett.106.153001

[14] Ho, C.M. (2011) Physics Letters B, 702, 398-401. https://doi.org/10.1016/j.physletb.2011.07.035

[15] Parkin, S., Knoner, G., Singer, W., Nieminen, T.A., Heckenberg, N.R. and Rubinsztein-Dunlop, H. (2007) Optical Torque on Microscopic Objects. In: Berns, M.W. and Greulich, K.O., Eds., Laser Manipulation of Cells and Tissues, Academic Press, Cambridge, 525-561. https://doi.org/10.1016/S0091-679X(06)82019-4

[16] Colbeck, R. and Renner, R. (2011) Nature Communications, $2,5$. https://doi.org/10.1038/ncomms1416

[17] Bell, J.S. (1964) Physics, 1, 195-200. https://doi.org/10.1103/PhysicsPhysiqueFizika.1.195

[18] Leggett, A. (2003) Foundations of Physics, 33, 1469-1493. https://doi.org/10.1023/A:1026096313729

[19] Groblacher, S., Paterek, T., Kaltenbaek, R., Brukner, C., Zukowski, M., Aspelmeyer, M., et al. (2007) Nature, 446, 871-875. https://doi.org/10.1038/nature05677

[20] De Zela, F. (2008) Journal of Physics A: Mathematical and Theoretical, 41, Article ID: 505301. https://doi.org/10.1088/1751-8113/41/50/505301

[21] De Broglie, L. (1925) Annales de Physique, 3, 3-109. http://tel.archives-ouvertes.fr/docs/00/04/70/78/PDF/tel-00006807.pdf https://doi.org/10.1051/anphys/192510030022

[22] Bohm, D. and Bub, J. (1966) Reviews of Modern Physics, 38, 453-469. https://doi.org/10.1103/RevModPhys.38.453

[23] Holland, P.R. (1993) Physics Reports, 224, 95-150. https://doi.org/10.1016/0370-1573(93)90095-U

[24] Medina, R. (1999) Annales de la Fondation Louis de Broglie, 24, 129-157.

[25] Egg, M. (2013) Foundations of Physics, 43, 872-880. https://doi.org/10.1007/s10701-013-9723-7

[26] Pons, D.J., Pons, A.D. and Pons, A.J. (2017) Journal of Modern Physics, 8, 1257-1274. https://doi.org/10.4236/jmp.2017.88082

[27] Pons, D.J. (2015) Internal Structure of the Electron (Image Licence Creative Commons Attribution 4.0). Wikimedia Commons, (Creative Commons Attribution 4.0 International License). https://commons.wikimedia.org/wiki/File:Internal structure of the electron.jpg

[28] Pons, D.J. (2015) Internal Structure of the Photon (Image Licence Creative Commons Attribution 4.0). Wikimedia Commons, Creative Commons Attribution 4.0 International License.

https://commons.wikimedia.org/wiki/File:Internal structure of the photon.jpg 
[29] Pons, D.J. (2015) Applied Physics Research, 7, 14-26. https://doi.org/10.5539/apr.v7n4p24

[30] Pons, D.J., Pons, A.D. and Pons, A.J. (2014) Applied Physics Research, 6, 50-63. https://doi.org/10.5539/apr.v6n3p50

[31] Pons, D.J., Pons, A.D. and Pons, A.J. (2015) Applied Physics Research, 7, 1-11. https://doi.org/10.5539/apr.v7n1p1

[32] Pons, D.J., Pons, A.D. and Pons, A.J. (2015) Applied Physics Research, 7, 1-13. https://doi.org/10.5539/apr.v7n2p1

[33] Pons, D.J., Pons, A.D. and Pons, A.J. (2014) Applied Physics Research, 6, 28-46. https://doi.org/10.5539/apr.v6n2p28

[34] Pons, D.J., Pons, A.D. and Pons, A.J. (2015) Journal of Nuclear and Particle Physics, 5, 58-69.

[35] Pons, D.J., Pons, A.D. and Pons, A.J. (2016) Journal of Modern Physics, 7, 1049-1067. https://doi.org/10.4236/jmp.2016.710094

[36] Pons, D.J., Pons, A.D. and Pons, A.J. (2015) Physics Research International, 2015, Article ID: 651361. https://doi.org/10.1155/2015/651361

[37] Pons, D.J., Pons, A.D. and Pons, A.J. (2013) Applied Physics Research, 5, 145-174. https://doi.org/10.5539/apr.v5n6p145

[38] Pons, D.J., Pons, A.D. and Pons, A.J. (2014) Journal of Modern Physics, 5, 1980-1994. https://doi.org/10.4236/jmp.2014.517193

[39] Pons, D.J., Pons, A.D. and Pons, A.J. (2016) Journal of Modern Physics, 7, 1277-1295. https://doi.org/10.4236/jmp.2016.710113

[40] Pons, D.J. and Pons, A.D. (2013) The Open Astronomy Journal, 6, 77-89. https://doi.org/10.2174/1874381101306010077

[41] Pons, D.J., Pons, A.D. and Pons, A.J. (2013) Applied Physics Research, 5, 23-47. https://doi.org/10.5539/apr.v5n6p23

[42] Pons, D.J., Pons, A.D. and Pons, A.J. (2016) Applied Physics Research, 8, 111-121. https://doi.org/10.5539/apr.v8n3p111

[43] Pons, D.J., Pons, A.D. and Pons, A.J. (2018) Journal of Modern Physics, 9, 500-523. https://doi.org/10.4236/jmp.2018.93035

[44] Pons, D.J., Pons, A.D. and Pons, A.J. (2013) Applied Physics Research, 5, 107-126. https://doi.org/10.5539/apr.v5n5107

[45] Pons, D.J., Pons, A.D. and Pons, A.J. (2014) Physics Essays, 27, 26-35. https://doi.org/10.4006/0836-1398-27.1.26

[46] Pons, D.J., Pons, A.D. and Pons, A.J. (2013) Proton-Neutron Bonds in Nuclides: Cis and Trans-Phasic Assembly with the Synchronous Interaction.

[47] Rutherford, E. (1911) Philosophical Magazine, 6, 669-688. https://doi.org/10.1080/14786435.2011.617037

[48] Dirac, P.A.M. (1962) Royal Society-Proceedings Series A, 268, 57-67. https://doi.org/10.1098/rspa.1962.0124

[49] Schwinger, J. (1948) Physical Review, 73, 416-417. http://journals.aps.org/pr/abstract/10.1103/PhysRev.73.416 https://doi.org/10.1103/PhysRev.73.416

[50] Miller, J.P., de Rafael, E. and Roberts, B.L. (2007) Reports on Progress in Physics, 70, 795. https://arxiv.org/abs/hep-ph/0703049 https://doi.org/10.1088/0034-4885/70/5/R03 
[51] Einstein, A., Podolsky, B. and Rosen, N. (1935) Physical Review, 47, 777. http://link.aps.org/doi/10.1103/PhysRev.47.777 https://doi.org/10.1103/PhysRev.47.777

[52] Pons, D.J., Pons, A.D. and Pons, A.J. (2015) Applied Physics Research, 7, 18-29. https://doi.org/10.5539/apr.v7n3p18

[53] Pons, D.J., Pons, A.D., Pons, A.M. and Pons, A.J. (2011) Special States of Matter. http://vixra.org/abs/1104.0025 